\title{
The 3D printing development study based on co-word analysis and multivariate statistics
}

\author{
Jinfeng Wang ${ }^{1, a}$, Luyao Zhang ${ }^{2, b}$, Peng Liu ${ }^{3, c}$ \\ ${ }^{1-4}$ Zhengzhou university of engineering management, Henan Zhengzhou \\ ajinfengwang@zzu.edu.cn, bzhangluyao723@126.com
}

Keywords: 3D printing, co-word analysis, Winisis, SPSS19.0

\begin{abstract}
This paper makes CNKI database the data source, baed on making 3D printing as the research theme for the literature among the years 2000-2014, do the multivariate statistical analysis on the basis of co-word analysis to reveal the status of current research in the field of domestic 3D printing, to acknowledge and grasp the main research focus, devolopment context and evolution process of the $3 \mathrm{D}$ industry in time, which providing a reasonable reference for the subsequent research.
\end{abstract}

\section{Introduction}

3D printing is a new technology which develop rapidly in manufacturing field in recent year, known as "manufacturing technology of the industrial revolution significance". As a new comprehensive technology, it combines mathematical modeling, intelligent information manufacturing, mechanical design, and many many frontier technology knowledge.

At present, the domestic analysis for developments in the field of emerging technical is mainly concentrated in the following aspects:(1) To predict the future development from the perspective of technical performance, such as Li Xiaoli around seven classes 3D printing technology, analyze the global business condition and range of application areas, points out that the industry pattern of traditional industries will be to intelligent manufacturing ${ }^{[1]}$;(2)Through summarizing technology development stage characteristics and influence factors, then analyze and judge the future prospect, such as Wang Zhonghong by analyzing the development of 3D printing process to predict the future trend of the technology development, and put forward some suggestion for its future development from the aspects of development strategy and policy ${ }^{[2]}$. In view of this, this paper mines 3D printing research hot spots through the relationship between the key words in literature, specifically on the basis of the literature with "3D printing" as the theme since 2000 to now, and conduct multivariate statistical analysis on the basis of common word analysis.

\section{Keywords filtering Based on the co-word analysis}

Co-word analysis belong to the basic method of content analysis, its theoretical basis is counting the number of occurrences of a word in the same literature, on the basis of it , these words are hierarchical clustering to reveal the affinity-disaffinity relationship between the word and the word, and analyze the structure change of the theme they represent, use the graphical and numerical more intuitive to show complex co-word network relationship between objects.

\section{Literature summary and keywords processing}

With "3D printing" as the theme before 2011, the number of post has been in a low level, in 2012 and 2013, entered the stage of sharply rising,154 in 2012, and 1265 from 2013 to present, the annual post and the total trend diagram is shown in Figure 1.

On the basis of literaturequantity statistics, summary and further preprocessing the keywords in the literature, mainly includes roughly removing the keywords which is out of the subject, and entry Winisis on keywords frequency statistics in accordance with the name of the article, the author, unit, published journals, publication date, cited times, category, key words, abstract and so on ${ }^{[3]}$. The article rank the keywords according to frequency high and low, select high frequency words as analysis object, finally confirm 30 keywords by the word frequency statistics, specific see Table 1. 


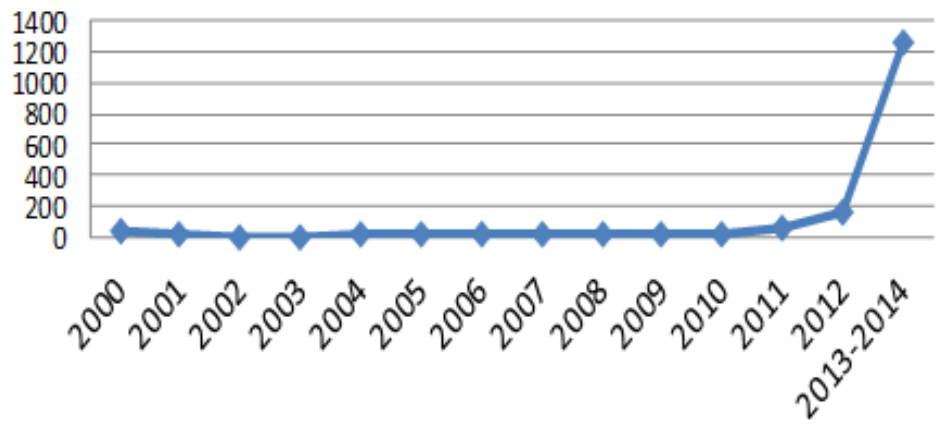

Figure 1 the overall trends of the number of research articlesrelated to 3D printing

Table1 Key words and word frequency of 3D printing field of research

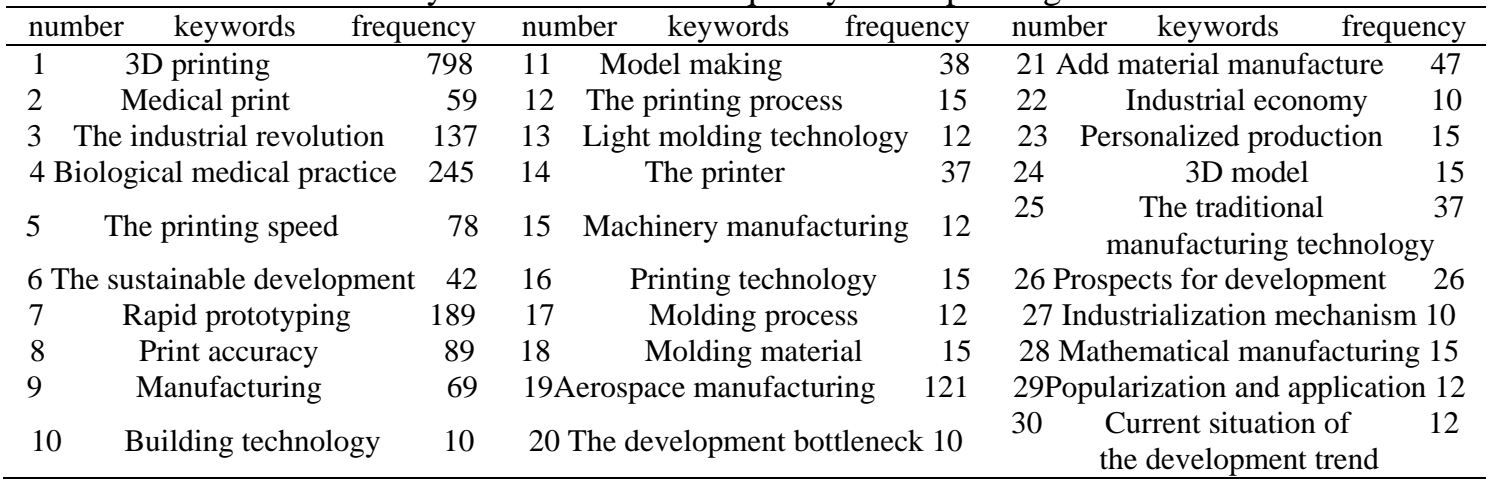

\section{Determine keywords matrix based on Co-word analysis}

1) Build word matrix

In the self-built database, has selected 30 keywords for a total of two word retrieval, the purpose is to statistics in 1422, at the same time, the number of occurrences of in the paper to form a common word $30 \times 30$ matrix, the matrix of the data are shown in Table 2. Formed on the basis of the total word matrix (including correlation matrices, similarity matrix and dissimilarity matrix), in order to use next.

Table 2 3D printing field keyword correlation matrix

\begin{tabular}{c|cccccccccc}
\hline No. & $\mathbf{1 1}$ & $\mathbf{4}$ & $\mathbf{7}$ & $\mathbf{5}$ & $\mathbf{6}$ & $\mathbf{3}$ & $\mathbf{8}$ & $\mathbf{2 1}$ & $\mathbf{9}$ & $\mathbf{1}$ \\
\hline $\mathbf{1 1}$ & 0 & 0 & 21 & 0 & 13 & 7 & 0 & 15 & 12 & 18 \\
$\mathbf{4}$ & 0 & 0 & 41 & 2 & 22 & 20 & 0 & 32 & 28 & 6 \\
$\mathbf{7}$ & 21 & 41 & 0 & 3 & 6 & 0 & 3 & 6 & 2 & 4 \\
$\mathbf{5}$ & 0 & 2 & 3 & 0 & 0 & 6 & 0 & 0 & 6 & 0 \\
$\mathbf{6}$ & 13 & 22 & 6 & 0 & 0 & 0 & 0 & 6 & 0 & 3 \\
$\mathbf{3}$ & 7 & 20 & 0 & 6 & 0 & 0 & 12 & 0 & 0 & 0 \\
$\mathbf{8}$ & 0 & 0 & 3 & 0 & 0 & 12 & 0 & 0 & 3 & 0 \\
$\mathbf{2 1}$ & 15 & 32 & 6 & 0 & 6 & 0 & 0 & 0 & 0 & 6 \\
$\mathbf{9}$ & 12 & 28 & 2 & 6 & 0 & 0 & 3 & 0 & 0 & 5 \\
$\mathbf{1}$ & 18 & 6 & 4 & 0 & 3 & 0 & 0 & 6 & 5 & 0 \\
\hline
\end{tabular}

2) Build similar matrix

To eliminate the co-occurrence frequency difference in the effects on the results of the analysis, the selection of coefficient transform correlation matrix for similar matrix, the calculation formula is: $O_{i j}=\frac{K_{i j}}{\sqrt{K_{i} \times K_{j}}}, K_{i}, K_{j}$ respectively represent the number of word frequency of keywords $\mathrm{i}$ and $\mathrm{j}$, $\mathrm{K}_{\mathrm{ij}}$ said keywords $K_{\mathrm{i}}$ and $K_{j}$ common word count. To calculate the similarity matrix.

3) Dissimilarity matrix

Because of the zero value of correlation matrix is more, easy to cause big error, for the convenience of further processing, use 1 and similar matrix data by subtracting,, you have two keywords dissimilarity degree of dissimilarity matrix.

\section{Multivariate statistical analysis of Keywords frequency}

According to the different research topic and purpose, need to take the corresponding statistical method to reveal hidden information in common word matrix. Usually methods including factor 
analysis, cluster analysis and multidimensional scaling analysis, etc.

\section{The high frequency keywords analysis}

Factor analysis was carried out on the common word correlation matrix in the matrix, its purpose is using as less as possible factor to describe the relationship between the indicators, the basic idea is to group the variables of study object according to the correlation, make the correlation among the variables within the same group is higher, the correlation of different set of variables is $\operatorname{low}^{[4]}$. The 30 variables' common degree in the factor analysis of this article is over $60 \%$, eigenvalues which are greater than 1 are three in total, the specific data analysis results are shown in Table 3.

Table 3 Factor and the information

\begin{tabular}{ccccccc}
\cline { 2 - 6 } & \multicolumn{3}{c}{ The initial eigenvalue } & \multicolumn{4}{c}{ Extraction of sum of squares loaded } \\
\cline { 2 - 7 } 1 & total & variance \% & cumulative \% & total & variance \% cumulative \% \\
\cline { 2 - 7 } & 5.630 & 56.305 & 56.305 & 5.630 & 56.305 & 56.305 \\
2 & 1.718 & 17.181 & 73.486 & 1.718 & 17.181 & 73.486 \\
3 & 1.425 & 14.255 & 87.740 & 1.425 & 14.255 & 87.740 \\
4 & .724 & 7.240 & 94.980 & & & \\
5 & .244 & 2.437 & 97.417 & & & \\
6 & .146 & 1.460 & 98.877 & & & \\
7 & .055 & .554 & 99.430 & & & \\
8 & .036 & .358 & 99.788 & & \\
9 & .021 & .212 & 100.000 & & & \\
10 & $-3.692 \mathrm{E}-18$ & $-3.692 \mathrm{E}-17$ & 100.000 & & & \\
\hline
\end{tabular}

In addition, Factor cumulative variance contribution rate in the factor analysis results is $87.74 \%$ (>50\%), each factor of the reliability and validity (different validity and convergent validity) has passed the inspection. At the same time use data processing results and factor of gravel chart of figure 3 for reference, the first three pivot can reach the ideal state and was chosen for public factor, that is, 30 variables are extracted as the three main factors to explain, mainly from the three aspects described through analyzing the research status of 3D printing.

\section{Research in the field of cluster analysis}

Based on the results of factor analysis, conduct clustering analysis on the similarity matrix from the treated Ochiia coefficient. In this paper, we use the method of system clustering, form three parts of the main research contents of the research in the field of 3D printing, summarized for 3d printing development process , 3D printing specific development application and problems faced in the development of 3D printing, specific clustering results as shown in Figure2.

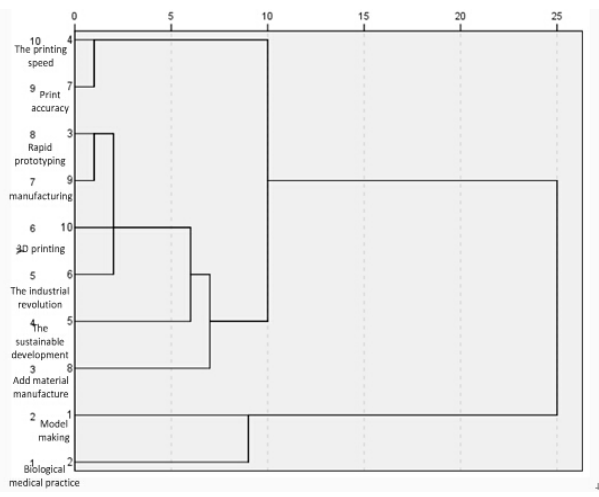

Figure2 Cluster analysis tree

\section{Summary}

\section{The development process and the future trend of 3D printing}

Based on the data analysis above, in combination with other keywords associated with 3D printing, study the present development situation and future development trend for further discussion.3D printing field related keywords to some extent reflects the evolution process of the field, Table4 has a statistics about landmark events.

Table 4 3D printing related signature time statistics ${ }^{[5]}$

2005 Z Croporatio released first high precision color adding machine in the world.

2008 Reference published the first edition, code-named "Darwin", can print their own 50\% components, only a box the size of the volume.

2008 San Francisco customized all the parts out of the prosthesis by adding a company manufacturing technology for the first time.

2009 Organovo in the United States use add manufacturing technology to create artificial blood vessels for the first time.

2011 The engineer in the university of Southampton produced the first drone made by the 3D printing. 
2012

The size of the global 3D printing market was \$2.204 billion,year-on-year growth of 28.6\%; Time magazine announced the best invention in 2012, loaded 3D printers.

2013 3D printing industry for the first time to was included in the national high technology (China) research and development program (863 program)

\section{The application range of the 3D printing}

It is not hard to find 3D printing has become the effective means in modern model and parts manufacturing during the process of keywords analysis in the literature, and has been widely used in the aerospace, engineering, biological medicine, teaching and research, and other fields

Bio-medical areas: domestic part of the hospital has successfully using 3D printing technology to provide patients with custom teeth and bone substitutes and the performance of bionic implants in the body; Industrial manufacturing areas: 3D printing technology has been widely used in conceptual design, mould making, model design, etc; Cultural creativity and digital entertainment: 3D printing can be used as expression vector of complex structure,special materials artistic; Aerospace, national defense fields: can directly manufacture he parts with complex shape, subtle size, special special performance.

\section{The challenge for the development of 3D printing}

In general, 3D printing industry in China is in the initial stage, rapid development as well as having a series of technical problems, mainly summarized as follows:

(1) Molding speed: Printing speed and efficiency is the problems of the 3D technology in the process of rapid development to be settled urgently, for now, 3D printers use more liquid resin material, the technology's print speed is only a few millimeters per second, then the new nano precision 3D printers and dynamic optical projection stereo lithography sharply improved the speed of 3D printing to print, but there are still a lot of room to improve.

(2) The printing material: Material is one of the key factor restricting the application and development of 3D printing technology, In simple terms, the demand to develop new materials mainly contains two levels, not only need the deep research on the structure of existing material, process, characteristics, clarify the advantages and limitations, also need to develop new test technique and method to expand the scope of available materials ${ }^{[6]}$.

(3) The quality of printing: In terms of print quality, for printing is the process of sintering rather than a full meltdown, has not yet to fully meet the requirements of material properties,hasn't even reach the traditional manufacturing level, so the precision of the rapid prototyping parts and quality can not meet the requirement of the present use mostly, cannot serve as the functional components, can only make the prototype. To sum up, the precision of 3D printing products and quality improvements still has great space for development.

\section{References}

[1] Li Xiaoli,Ma Jianxiong,Li Ping. 3D printing technology and application trends [J]. Process Automation Instrumentation,2014,35(1):1-5.

[2] Wang Zhonghong,Li Yangfan,Zhang Manyin. China's present situation and development of 3D printing industry [J]. Economic Review,2013, (1):90-93.

[3] Fu Yao,Yang Pan. The progress correlation data in China is based on the analysis of the common words [J]. Researches In Library Science,2013,04:18-24.

[4] Fan Shaoping,Li Yingying,Zhang Zhiqiang. Word analysis and study of literature measurement analysis at home and abroad [J]. Journal of Information,2013,09:104-109.

[5] Zhang Jide. China's 3D printing technology development present situation, problems and countermeasures - from catic juki (600765) think of successive harden [J]. Friends of Accounting,2013,12:56-60.

[6] Wan Wentao,Liu Yanhua. 3D printing manufacturing technology development trend and the influence of the structure transformation to our country [J]. Science and Technology Management Research,2014,34(6):22-25,30. 